\title{
Bits Security of the Elliptic Curve Diffie-Hellman Secret Keys
}

\author{
Dimitar Jetchev ${ }^{1}$ and Ramarathnam Venkatesan ${ }^{2,3}$ \\ ${ }^{1}$ Dept. of Mathematics, University of California at Berkeley, Berkeley, CA 94720 \\ jetchev@math. berkeley.edu \\ ${ }^{2}$ Microsoft Research, One Microsoft Way, Redmond WA 98052 \\ 3 Microsoft Research India Private Limited, "Scientia", No:196/36, \\ 2nd Main Road, Sadashivnagar, Bangalore - 560080, India \\ venkie@microsoft.com
}

\begin{abstract}
We show that the least significant bits (LSB) of the elliptic curve Diffie-Hellman secret keys are hardcore. More precisely, we prove that if one can efficiently predict the LSB with non-negligible advantage on a polynomial fraction of all the curves defined over a given finite field $\mathbb{F}_{p}$, then with polynomial factor overhead, one can compute the entire Diffie-Hellman secret on a polynomial fraction of all the curves over the same finite field. Our approach is based on random self-reducibility (assuming GRH) of the Diffie-Hellman problem among elliptic curves of the same order. As a part of the argument, we prove a refinement of $\mathrm{H}$. W. Lenstra's lower bounds on the sizes of the isogeny classes of elliptic curves, which may be of independent interest.
\end{abstract}

\section{Introduction}

The Diffie-Hellman protocol for key exchange [16 is based on the hardness of computing the function $\mathrm{DH}_{g}\left(g^{u}, g^{v}\right)=g^{u v}$, where $g$ is a fixed generator of the multiplicative group of a finite field $\mathbb{F}_{p}$, and $1 \leq u, v \leq p-1$ are integers. A natural question is whether one can compute some of the bits of $g^{u v}$ given $g, g^{u}, g^{v}$. It is unknown if predicting partial information with significant advantage over a random guess will lead to a compromise of the Diffie-Hellman function. Boneh and Venkatesan 2, 25, have shown that if one is able to compute (in time polynomial in $\log p)$ the $5 \sqrt{\log p}$ most significant bits of $g^{u v}$ for every input $\left(g^{u}, g^{v}\right)$ then one can compute (in polynomial time) the entire shared secret key $g^{u v}$. For motivation, note that $g^{u v}$ may be 1024 bits long, but one may want to use the least significant 128 bits of $g^{u v}$ as a block cipher key. Thus, it is important to know that partial information is not computable or predictable with any significant advantage over a random guess. Another motivation stems from the fact that the methods used in 2 suggest attacks on cryptographic systems that reveal some information about $g^{u v}$ to the attacker [8, [10, [18, 19, [20, 24, 26.

D. Wagner (Ed.): CRYPTO 2008, LNCS 5157, pp. 75 92, 2008.

(C) International Association for Cryptologic Research 2008 
The analogous problem for elliptic curves studies the bit security of the following function:

Diffie-Hellman function: Let $E$ be an elliptic curve over $\mathbb{F}_{p}$ and let $P \in E$ be a point of prime order $q$. We define the Diffie-Hellman function as

$$
\mathrm{DH}_{E, P}(u P, v P)=u v P
$$

where $1 \leq u, v \leq q$ are integers. Moreover, we refer to the triple $(P, u P, v P)$ as a Diffie-Hellman triple for $E$.

For simplicity, we restrict ourselves to short Weierstrass equations (models) of $E$, i.e., models of the form $y^{2}=x^{3}+a x+b$ with $a, b \in \mathbb{F}_{p}$ and $4 a^{3}+27 b^{2} \neq 0$. By abuse of common terminology, an elliptic curve for us will be an $\mathbb{F}_{p}$-isomorphism class of short Weierstrass equations. It is not hard to see that the complexity of the Diffie-Hellman function is independent of the choice of the short Weierstrass equation for the elliptic curve $E$ over $\mathbb{F}_{p}$. Indeed, given two different models $W$ and $W^{\prime}$ for $E$ over $\mathbb{F}_{p}$ and an explicit isomorphism $\varphi: W \rightarrow W^{\prime}$ and its inverse $\varphi^{-1}: W^{\prime} \rightarrow W$, a Diffie-Hellman triple $(P, u P, v P)$ on $W$ is mapped to a Diffie-Hellman triple $(\varphi(P), u \varphi(P), v \varphi(P))$ on $W^{\prime}$ and therefore, if one can compute $u v \varphi(P)$, one would know $u v P$. Yet, if one wants to formalize the notion of security of single bits of the Diffie-Hellman function, one needs to choose a short Weierstrass model (it is not necessarily true any more that if one knows one bit of the Diffie-Hellman secret $u v \varphi(P)$ on $W^{\prime}$ then one can compute the corresponding bit of $u v P$ on $W$ ).

Boneh and Shparlinski [1] have reduced (in time polynomial in $\log p$ ) the Diffie-Hellman problem on an elliptic curve $E$ to the problem of predicting the LSB of the secret key $u v P$ with non-negligible advantage over a random guess on a polynomial fraction of all short Weierstrass models for $E$. Alternatively, if one looks for a polynomial time reduction of the Diffie-Hellman problem to the problem of predicting partial information on the same short Weierstrass model $W$, some results have been established using Gröbner bases [12].

A more general and natural situation would be to consider an oracle $\mathcal{A}$ that predicts the least significant bit of the Diffie-Hellman secret key for short Weierstrass models $W$ chosen from a non-negligible subset $G$ (i.e., from a $(\log p)^{O(1)}$ fraction) of all the short Weierstrass equations over $\mathbb{F}_{p}$ and arbitrary DiffieHellman triples on these models. Here, one encounters extra challenges. First, the set $G$ may be distributed arbitrarily over all (exponentially many in $\log p$ ) isogeny classes of short Weierstrass models, where each isogeny class contains exponentially many isomorphism classes of short Weierstrass models, with each isomorphism class containing exponentially many short Weierstrass models. Second, relating the difficulty of computing the Diffie-Hellman function within each isogeny class is itself a nontrivial task: having an explicit (computable in time polynomial in $\log p$ ) isogeny from an elliptic curve $E$ to another curve $E^{\prime}$ in the same class would achieve this task. By Tate's isogeny theorem [28, such a map exists if and only if $E$ and $E^{\prime}$ have the same number of points( $E$ and $E^{\prime}$ are said to be isogenous). Yet, such an isogeny can have large degree and it can take 
superpolynomial number of steps to compute it. Typically, isogeny computations are used in attacks such as the Weil descent attack [3], [7].

We show that such an oracle $\mathcal{A}$ is unlikely to exist by proving that its existence would imply the existence of a set $S$ of polynomial (in $\log p$ ) fraction of all elliptic curves over $\mathbb{F}_{p}$ so that one can solve the Diffie-Hellman problem for every $E \in S$ and every Diffie-Hellman triple $(P, u P, v P)$ for $E$. This is based on random self-reducibility among elliptic curves, which was first studied in[13]; by Tate's theorem achieving this via algebraic maps (isogenies) is possible only among those curves that have the same order (or trace). Thus our focus here is to identify the values of the trace for which the self-reducibility is applicable. This allows us to use Boneh-Shparlinski hard core bit result on isomorphism classes and enlarge the set of curves where it is applicable. For example, if on a specific isomorphism class their oracle algorithm does not apply, our random walk can (with a good probability) link it to another class where it applies. To show the hard core bit theorem for all the curves, one may consider the analysis based only on isomorphism classes, but the associated hardness assumption is clear and natural when restricted isogeny classes (in view of Tate's theorem). It will be interesting to see if one can develop new attacks, similar to the ones mentioned earlier for the finite field case. We remark that hard core bit theorems for finite field Diffie-Hellman function remain open and the best in this case is computing one bit (without error) is hard, if the generator is small [2].

\section{Notation and Preliminaries}

Throughout, $p \geq 5$ will be a prime number and $\tilde{\varepsilon}>0$ will be a fixed real number. We will be considering the Diffie-Hellman problem for elliptic curves $E$ over $\mathbb{F}_{p}$ and triples $(P, u P, v P)$, where $P$ is a point of prime order $q>(\log p)^{2+\tilde{\varepsilon}}$ and $1 \leq u, v \leq q$ are integers. We make this assumption because an isogeny $\phi: E \rightarrow E^{\prime}$ of prime degree $\ell \leq(\log p)^{2+\tilde{\varepsilon}}$ will preserve the order of $P$ and this assumption will be necessary for what follows.

We say that an oracle $\mathcal{B}$ computes the Diffie-Hellman function for $E$ if for any point $P$ of prime order $q>(\log p)^{2+\tilde{\varepsilon}}$,

$$
\mathcal{B}(P, u P, v P)=u v P
$$

holds with probability at least $1-1 / p$ (here, the probability is taken over all possible choices of $u$ and $v$ ).

Moreover, if $z$ is a non-negative integer then $\operatorname{LSB}(z)$ will denote the least significant bit of $z$. To define the least significant bit of an element $x \in \mathbb{F}_{p}$, we first look at the identity map $\iota: \mathbb{F}_{p} \rightarrow \mathbb{Z} / p \mathbb{Z}$. If $0 \leq z \leq p-1$ is the unique integer whose image is $\iota(x)$, we define $\operatorname{LSB}(x)=\operatorname{LSB}(z)$. Also, if $Q \in E\left(\mathbb{F}_{p}\right)$ then $x(Q)$ and $y(Q)$ denote the $x$ - and $y$-coordinates of $Q$, respectively.

Finally, let $H=\{t \in \mathbb{Z}:|t| \leq 2 \sqrt{p}\}$ be the Hasse interval. For $t \in H$ one can write $t^{2}-4 p$ uniquely as $d_{t} c_{t}^{2}$, where $d_{t}<0$ is square-free and $c_{t}>0$. We call $c_{t}$ the conductor of $t$. 
Advantage: Let $\mathcal{A}$ be an algorithm that, given a short Weierstrass equation $W$ over $\mathbb{F}_{p}$, a point $P \in W\left(\mathbb{F}_{p}\right)$ of prime order $q>(\log p)^{2+\tilde{\varepsilon}}$ and two multiples $u P$ and $v P$ with $1 \leq u, v \leq q-1$, outputs a single bit. We define the advantage $\operatorname{Adv}_{W, P}(\mathcal{A})$ of $\mathcal{A}$ as

$$
\operatorname{Adv}_{W, P}(\mathcal{A}):=\left|\underset{u, v}{\operatorname{Pr}}[\mathcal{A}(P, u P, v P)=\operatorname{LSB}(x(u v P))]-\frac{1}{2}\right| .
$$

We say that $\mathcal{A}$ has an advantage $\varepsilon$ on $W$ if $\operatorname{Adv}_{W, P}(\mathcal{A})>\varepsilon$ holds for any point $P \in W\left(\mathbb{F}_{p}\right)$ of prime order $q>(\log p)^{2+\tilde{\varepsilon}}$.

\section{The Main Result}

For each prime $p$, let

$$
\Gamma_{p}=\left\{W_{a, b}:(a, b) \in \mathbb{F}_{p} \times \mathbb{F}_{p}, 4 a^{3}+27 b^{2} \neq 0\right\}
$$

be the set of all short Weierstrass equations and let $\Omega_{p}$ be the set of all elliptic curves over $\mathbb{F}_{p}$ (i.e., $\left.\Omega_{p}=\Gamma_{p} / \cong_{\mathbb{F}_{p}}\right)$. Let $\Omega_{p}^{(t)}$ and $\Gamma_{p}{ }^{(t)}$ denote the restriction to those curves with trace $t$.

Theorem 3.1. Assume the Generalized Riemann Hypothesis (GRH) and let $c>$ 0 be a fixed real. (a) For almost every $t$ in the Hasse interval, the Diffie-Hellman problem is random self reducible among the set of elliptic curves with trace $t$. (b) Given a subset $G \subset \Gamma_{p}$, such that $|G|=\delta\left|\Gamma_{p}\right|$ for some $0<\delta \leq 1$ with $1 / \delta=O\left((\log p)^{c}\right)$, assume that there exists $\varepsilon>0$ and an algorithm $\mathcal{A}$ running in time $t$ that takes as input a short Weierstrass model $W$ and a Diffie-Hellman triple $(P, u P, v P)$ and outputs a single bit. Assume that $\mathcal{A}$ satisfies the following property: for any $W \in G$ and any point $P$ of prime order $q>(\log p)^{2+\tilde{\varepsilon}}$ on $W$, $\operatorname{Adv}_{W, P}(\mathcal{A})>\varepsilon$. Then there exists a subset $S \subseteq \Omega_{p}$ satisfying

$$
\frac{\left|\Omega_{p}\right|}{|S|}=O_{c}\left((\log p)^{\frac{3(c+1)}{2}}(\log \log p)^{4}\right),
$$

and an algorithm $\mathcal{B}$ running in time $\left(\varepsilon^{-1} \log p\right)^{O(1)}$, such that $\mathcal{B}$ computes the entire Diffie-Hellman secret $\mathrm{DH}_{E, P}(u P, v P)$ for any $E \in S$ and any DiffieHellman triple $(P, u P, v P)$ for $E$ (Note that in the above displayed formula, the implied constant depends only on $c$ ). Moreover, these statements hold true with $\Omega_{p}$ and $\Gamma_{p}$ replaced by $\Omega_{p}^{(t)}$ and $\Gamma_{p}{ }^{(t)}$ for almost every value of the trace $t$.

Intuitively, (a) implies that an efficient algorithm for computing the Diffie-Hellman function in the average case would imply an efficient algorithm for the same function in the worst case (see Section 6 for the precise technical definition).

\section{Counting Elliptic Curves}

Let $p \geq 5$ be a prime and let $\Gamma_{p}=\left\{W_{a, b}:(a, b) \in \mathbb{F}_{p} \times \mathbb{F}_{p}, 4 a^{3}+27 b^{2} \neq 0\right\}$ be the set of all short Weierstrass equations over $\mathbb{F}_{p}$. Explicitly, $W_{a, b}$ is the short 
Weierstrass equation $y^{2}=x^{3}+a x+b$. Then $\left|\Gamma_{p}\right|=p(p-1)$ since the number of all pairs $(a, b)$, such that $4 a^{3}+27 b^{2}=0$ is equal to $p$. Indeed, any such pair is parameterized by $a=-3 c^{2}$ and $b=2 c^{3}$ for some $c \in \mathbb{F}_{p}$ and each such $c$ is uniquely determined from $(a, b)$.

\subsection{Isomorphism Classes}

Two short Weierstrass equations $W_{a, b}$ and $W_{a^{\prime}, b^{\prime}}$ are isomorphic over $\mathbb{F}_{p}$ if there exists an element $u \in \mathbb{F}_{p}^{\times}$, such that $a^{\prime}=u^{4} a$ and $b^{\prime}=u^{6} b$. To count the elliptic curves $E$ over $\mathbb{F}_{p}$, we observe that the number of short Weierstrass equations $W \in \Gamma_{p}$ for $E$ is exactly $\frac{p-1}{\# \operatorname{Aut}(E)}$. In particular, this gives us the formula

$$
\sum_{E} \frac{1}{\# \operatorname{Aut}(E)}=p
$$

where the sum is taken over all elliptic curves $E$ over $\mathbb{F}_{p}$.

\subsection{Isogeny Classes}

Tate's isogeny theorem states that two elliptic curves $E_{1}, E_{2}$ over $\mathbb{F}_{p}$ are isogenous if and only if $\# E_{1}\left(\mathbb{F}_{p}\right)=\# E_{2}\left(\mathbb{F}_{p}\right)$. For any elliptic curve $E_{/ \mathbb{F}_{p}}$ we have the Hasse bound $\left|p+1-\# E\left(\mathbb{F}_{p}\right)\right| \leq 2 \sqrt{p}$. For an integer $t \in H$ consider the isogeny class of short Weierstrass equations

$$
C_{t}=\left\{W_{a, b} \in \Gamma: \# W_{a, b}\left(\mathbb{F}_{p}\right)=p+1-t\right\} .
$$

Our goal is to provide upper and lower bounds on the size of $C_{t}$ for any $t \in H$. We show how to do this in the next two sections. A useful definition for what follows is the weighted cardinality:

Definition 4.1 (Weighted cardinality). Let $U$ be any set of elliptic curves over $\mathbb{F}_{p}$. We define the weighted cardinality to be the sum

$$
\#^{\prime} U=\sum_{E \in U} \frac{1}{\# \operatorname{Aut}(E)} .
$$

\subsection{Lenstra's Upper Bound}

Lemma 4.1. Let $\Sigma$ be a set of integers $t$ satisfying $|t| \leq 2 \sqrt{p}$. There exists an effectively computable constant $c_{u}$ (independent of $p$ ), such that

$$
\sum_{t \in \Sigma}\left|C_{t}\right| \leq c_{u}|\Sigma| p^{3 / 2}(\log p)(\log \log p)^{2} .
$$

Proof. By [15, Prop.1.9(a)], there exists an effective constant $c$, such that

$$
\#^{\prime}\left\{W \in \Gamma_{p}: 1+p-\# W\left(\mathbb{F}_{p}\right) \in \Sigma\right\} / \cong_{\mathbb{F}_{p}} \leq c|\Sigma| p^{1 / 2}(\log p)(\log \log p)^{2} .
$$

Now, the lemma is a consequence of the fact that the weight of an elliptic curve $E$ is $(\# \operatorname{Aut}(E))^{-1}$ (which is either $1 / 2,1 / 3$ or $1 / 6$ ) and that the isomorphism class corresponding to $E$ contains $\frac{p-1}{\# \operatorname{Aut}(E)}$ short Weierstrass equations. 


\subsection{Refining Lenstra's Lower Bound}

We need a simple refinement of the lower bound established by Lenstra in [15. Prop.1.9(b)] on the size of a collection of isogeny classes.

If $|t| \leq 2 \sqrt{p}$, the weighted number of elliptic curves over $\mathbb{F}_{p}$ whose trace of Frobenius is $t$ is equal to the Kronecker class number $H\left(t^{2}-4 p\right)$ (see [4, [15, pp.654-655]). For a fixed integer $\Delta<0, \Delta \equiv 0,1 \bmod 4$, the Kronecker class number $H(\Delta)$ is the weighted number of equivalence classes of binary quadratic forms of discriminant $\Delta$ (the weight of a quadratic form is defined to be inverse of the number of automorphisms of the form). Let $\Delta_{0}$ be the fundamental discriminant associated with $\Delta$ and let $\chi_{0}$ be the quadratic character associated to $\Delta_{0}$. Using an analytic class number formula, one expresses $H(\Delta)$ in terms of the special value $L\left(1, \chi_{0}\right)$ of the $L$-function of the character $\chi_{0}$ and the discriminant $\Delta$. Thus, a lower bound for $H(\Delta)$ would follow from a lower bound on the special value of the above $L$-function. The following result is proved in [15, Prop.1.8]:

Lemma 4.2. (i) There exists an effectively computable positive constant $c_{0}$, such that for each $z \in \mathbb{Z}_{>0}$, there exists $\Delta^{*}=\Delta^{*}(z)$, such that

$$
H(\Delta) \geq c_{0} \frac{|\Delta|^{1 / 2}}{\log z},
$$

for each $\Delta$ which satisfies $|\Delta| \leq z, \Delta<0, \Delta \equiv 0,1 \bmod 4$ and $\Delta_{0} \neq \Delta^{*}$. (ii) Assume the Generalized Riemann Hypothesis. There exists an effectively computable constant $c_{0}^{\prime}>0$, such that for each $z \in \mathbb{Z}_{>0}$

$$
H(\Delta) \geq c_{0}^{\prime} \frac{|\Delta|^{1 / 2}}{\log \log z},
$$

for each $\Delta$ which satisfies $|\Delta| \leq z, \Delta<0$ and $\Delta \equiv 0,1 \bmod 4$.

The following refinement of Lenstra's Proposition 1.9(b) is necessary for our argument:

Proposition 4.1. Let $0<\mu<1$ and let $\Sigma$ be a set of integers $t$ satisfying $|t| \leq 2 \sqrt{p}(1-\mu)$. Let

$$
w_{\Sigma}=\#^{\prime}\left\{E: E \text { elliptic curve over } \mathbb{F}_{p}, 1+p-\# E\left(\mathbb{F}_{p}\right) \in \Sigma\right\} / \cong \mathbb{F}_{p},
$$

be the weighted cardinality of the short Weierstrass equations whose traces of Frobenius are in $\Sigma$.

(i) There exists an effectively computable constant $c_{1}>0$, such that

$$
w_{\Sigma} \geq c_{1}(|\Sigma|-2) \frac{\mu^{1 / 2} p^{1 / 2}}{\log p} .
$$

(ii) Assume the Generalized Riemann Hypothesis. Then there exists an effectively computable constant $c_{1}^{\prime}>0$, such that

$$
w_{\Sigma} \geq c_{1}^{\prime}|\Sigma| \frac{\mu^{1 / 2} p^{1 / 2}}{\log \log p} .
$$


Proof. One can express

$$
w_{\Sigma}=\sum_{t \in \Sigma} H\left(t^{2}-4 p\right)
$$

i) We apply Lemma 4.2 with $z=4 p$ to get that there exists a constant $c_{0}>0$, such that $H(\Delta) \geq c_{0} \frac{|\Delta|^{1 / 2}}{\log p}$ unless $\Delta_{0}=\Delta^{*}$. As in the proof of Lenstra's Proposition 1.9(b), there are at most two values of $t$ for which the fundamental discriminant of $t^{2}-4 p$ is equal to $\Delta^{*}$. Hence, it remains to estimate $\left|t^{2}-4 p\right|$ from below to obtain a lower estimate on $w_{\Sigma}$. But if $t \in \Sigma$ then

$$
\left|t^{2}-4 p\right| \geq 4 p-4 p(1-\mu)^{2}=8 \mu p-4 \mu^{2} p>8 \mu p-4 \mu p=4 \mu p .
$$

Thus, $\left|t^{2}-4 p\right|^{1 / 2} \geq 2 \mu^{1 / 2} p^{1 / 2}$. Hence, if $c_{1}=c_{0}$ then

$$
w_{\Sigma} \geq c_{1}(|\Sigma|-2) \frac{\mu^{1 / 2} p^{1 / 2}}{\log p} .
$$

ii) The second part follows similarly except that we use the lower bound under the Generalized Riemann Hypothesis for the Kronecker class number $H(\Delta)$ from Lemma 4.2(ii).

\section{$5 \quad$ Isogeny Graphs}

We recall a construction for isogeny graphs for ordinary elliptic curves [13. For an integer $t \in H$ consider the isogeny class $C_{t} \subset \Gamma_{p}$ of short Weierstrass equations over $\mathbb{F}_{p}$. Let $S_{t}=C_{t} / \sim$ be the corresponding isogeny class of elliptic curves (i.e., we identify two short Weierstrass equations $W_{a, b}, W_{a^{\prime}, b^{\prime}} \in C_{t}$ if they are isomorphic over $\mathbb{F}_{p}$ ).

Throughout the whole paper, an isogeny between two elliptic curves will always mean an isogeny defined over $\mathbb{F}_{p}$.

\subsection{Ordinary Isogeny Classes and Isogeny Volcanoes}

1. Ordinary isogeny classes. Suppose that $S_{t}$ is an isogeny class of ordinary elliptic curves. To understand the structure of $S_{t}$ one looks at the endomorphism rings of the elliptic curves inside $S_{t}$. For any curve $E \in S_{t}$, the endomorphism ring $\operatorname{End}(E)$ is an order in a quadratic imaginary field [27, §III.9]. Let $\pi: E \rightarrow E$ be the Frobenius endomorphism. The characteristic polynomial of $\pi$ is $X^{2}-t X+$ $p=0$, so we can regard $\pi$ as an algebraic integer. It only depends on the class $S_{t}$. The following theorem is proved in [14, §4.2] (see also [13, Thm.2.1])

Theorem 5.1 (Kohel). Let $E$ and $E^{\prime}$ be two elliptic curves over $\mathbb{F}_{p}$ that are isogenous over $\mathbb{F}_{p}$, let $K$ be the quadratic imaginary field $\operatorname{End}(E) \otimes \mathbb{Q}$ and $\mathcal{O}_{K}$ be the maximal order of $K$. 
1. We have $\mathbb{Z}[\pi] \subseteq \operatorname{End}(E) \subseteq \mathcal{O}_{K}$ and $\mathbb{Z}[\pi] \subseteq \operatorname{End}\left(E^{\prime}\right) \subseteq \mathcal{O}_{K}$.

2. The following are equivalent:

(a) $\operatorname{End}(E)=\operatorname{End}\left(E^{\prime}\right)$

(b) There exist isogenies $\phi: E \rightarrow E^{\prime}$ and $\psi: E^{\prime} \rightarrow E$ of relatively prime degree.

(c) $\left(\mathcal{O}_{K}: \operatorname{End}(E)\right)=\left(\mathcal{O}_{K}: \operatorname{End}\left(E^{\prime}\right)\right)$.

3. Let $\phi: E \rightarrow E^{\prime}$ be an isogeny from $E$ to $E^{\prime}$ of prime degree $\ell$ defined over $\mathbb{F}_{p}$. Then one of the three cases occurs: i) $\operatorname{End}(E)$ contains $\operatorname{End}\left(E^{\prime}\right)$ with index $\ell$; ii) $\operatorname{End}\left(E^{\prime}\right)$ contains $\operatorname{End}(E)$ with index $\ell$; iii) $\operatorname{End}\left(E^{\prime}\right)=\operatorname{End}(E)$.

4. Let $\ell$ be a prime that divides exactly one of $\left(\mathcal{O}_{K}: \operatorname{End}(E)\right)$ and $\left(\mathcal{O}_{K}\right.$ : $\left.\operatorname{End}\left(E^{\prime}\right)\right)$. Then every isogeny $\phi: E \rightarrow E^{\prime}$ has degree a multiple of $\ell$.

2. Isogeny volcanoes. A convenient visualization of the elliptic curves in an isogeny class in the ordinary case together with the isogenies between them is given by isogeny volcanoes [5], 14. The curves are represented in levels according to their endomorphism rings. Two curves $E_{1}$ and $E_{2}$ are in the same level if and only if $\operatorname{End}\left(E_{1}\right) \cong \operatorname{End}\left(E_{2}\right)$. Thus, every level corresponds to an order $\mathcal{O}$ in a fixed quadratic imaginary field $K$. The level corresponding to an order $\mathcal{O}$ is above the level corresponding to an order $\mathcal{O}^{\prime}$ if $\mathcal{O} \supsetneq \mathcal{O}^{\prime}$.

Following [5], 6] and [14], we distinguish among three types of isogenies $\phi$ : $E \rightarrow E^{\prime}$ of prime degree $\ell$ over $\mathbb{F}_{p}$ :

1. $\phi$ is horizontal if $\operatorname{End}(E)=\operatorname{End}\left(E^{\prime}\right)$;

2. $\phi$ is up if $\left(\operatorname{End}\left(E^{\prime}\right): \operatorname{End}(E)\right)=\ell$;

3. $\phi$ is down if $\left(\operatorname{End}(E): \operatorname{End}\left(E^{\prime}\right)\right)=\ell$.

One can compute the number of horizontal, up and down isogenies of a given prime degree coming out of a particular ordinary elliptic curve $E$ in terms of the degree and the Legendre symbol. The result (see [5, §2.1], [6, Thm.4] and [14, Ch.4, Prop.23]) is summarized in the following

Proposition 5.1. Let $E$ be an ordinary elliptic curve over $\mathbb{F}_{p}$, with endomorphism ring $\operatorname{End}(E)$ contained in the quadratic imaginary field $K$ with fundamental discriminant $-D<0$. Let $\ell$ be a prime different from $p$ and let $c_{\pi}=\left(\mathcal{O}_{K}\right.$ : $\mathbb{Z}[\pi])$ and $c_{E}=\left(\mathcal{O}_{K}: \operatorname{End}(E)\right)$. Then

1. Assume $\ell \nmid c_{E}$. Then there are exactly $1+\left(\frac{-D}{\ell}\right)$ horizontal isogenies $\phi$ : $E \rightarrow E^{\prime}$ of degree $\ell$ over $\mathbb{F}_{p}$.

(a) If $\ell \nmid c_{\pi}$, there are no other isogenies $E \rightarrow E^{\prime}$ of degree $\ell$ over $\mathbb{F}_{p}$.

(b) If $\ell \mid c_{\pi}$, there are $\ell-\left(\frac{-D}{\ell}\right)$ down isogenies of degree $\ell$ over $\mathbb{F}_{p}$.

2. Assume $\ell \mid c_{E}$. Then there is one up isogeny $E \rightarrow E^{\prime}$ of degree $\ell$ over $\mathbb{F}_{p}$.

(a) If $\ell \nmid \frac{c_{\pi}}{c_{E}}$ then there are no horizontal isogenies of degree $\ell$ over $\mathbb{F}_{p}$.

(b) If $\ell \mid \frac{c_{\pi}^{E}}{c_{E}}$ then there are $\ell$ down isogenies of degree $\ell$ over $\mathbb{F}_{p}$.

Finally, we say that two isomorphism classes of elliptic curves $E_{1}$ and $E_{2}$ in the same isogeny class belong to the same level of the isogeny volcano if and only if $c_{E_{1}}=c_{E_{2}}$. 


\subsection{Expander Graphs and a Rapid Mixing Lemma}

Let $k$ be a positive integer and let $\mathcal{I}$ be an infinite set of positive integers. Consider a sequence of graphs $\left\{G_{h}\right\}_{h \in \mathcal{I}}$, each of which is $k$-regular and connected, such that $G_{h}$ has $h$ vertices. Let $A_{h}$ be the adjacency matrix of $G_{h}$. Since $G_{h}$ is $k$-regular, the vector $v_{h}$ consisting of 1's in each coordinate is an eigenvectors for $A_{h}$ with eigenvalue $\lambda_{\text {triv }}=k$ and any other eigenvalue $\lambda$ of $A_{h}$ satisfies $|\lambda| \leq k$. We refer to the eigenvalue $\lambda_{\text {triv }}$ as the trivial eigenvalue. Furthermore, since $G_{h}$ is connected, the eigenvalue $k$ has multiplicity one.

Definition 5.1. The sequence $\left\{G_{h}\right\}_{h \in \mathcal{I}}$ is called a sequence of expander graphs if there exists a constant $0<\nu<1$, such that for any $h$ and any eigenvalue $\lambda \neq \lambda_{\text {triv }}$ of $A_{h},|\lambda| \leq \nu \lambda_{\text {triv }}$.

The main application of expander graphs is to prove the rapid mixing of random walks provided we have a good upper bound on the spectral gap $\nu$. The property is summarized in the following proposition which will be used in our particular application (see [13, Prop.3.1] for a proof):

Proposition 5.2. Let $G$ be a $k$-regular graph with $h$ vertices. Assume that every eigenvalue $\lambda \neq \lambda_{\text {triv }}$ of $G$ satisfies the bound $|\lambda| \leq \nu \lambda_{\text {triv }}$ for some $0<\nu<1$. Let $S$ be a set of vertices of $G$ and let $x$ be any vertex of $G$. Then a random walk of length at least $\frac{\log \left(\frac{2 h}{|S|^{1 / 2}}\right)}{\log \left(\nu^{-1}\right)}$ starting at $x$ will land in $S$ with probability at least $\frac{|S|}{2 h}$.

\subsection{Isogeny Graphs in the Ordinary Case}

Fix an isogeny class $C_{t}$ of short Weierstrass equations for some $t \in H$ and the corresponding set $S_{t}$ of elliptic curves. Following [13, §2.1] we define an isogeny graph to be a graph $\mathcal{G}$ whose vertices are all the elements of $S_{t}$ that belong to a fixed level of the isogeny volcano for $S_{t}$.

Let $E_{1}, E_{2} \in S_{t}$. Two isogenies $\phi: E_{1} \rightarrow E_{2}$ and $\phi^{\prime}: E_{1} \rightarrow E_{2}$ are said to be equivalent if there exists an automorphism $\alpha \in \operatorname{Aut}\left(E_{2}\right)$, such that $\phi^{\prime}=\alpha \phi$ (see also [9, Prop.2.3]). The edges of the graph are equivalence classes of horizontal isogenies that have prime degrees at most $(\log p)^{2+\tilde{\varepsilon}}$. The degree bound is chosen in such a way that it is small enough to allow the isogenies to be computed and large enough to allow the graph to be connected and to have rapid mixing properties.

The graph $\mathcal{G}$ is known to be isomorphic to a graph $\mathcal{H}$ whose vertices are elliptic curves $\mathbb{C} / \mathfrak{a}$ with complex multiplication by the order $\mathcal{O}$ corresponding to the level for the graph $\mathcal{G}$ in the isogeny volcano (here, $\mathfrak{a} \subset \mathcal{O}$ is an ideal) and whose edges are isogenies of the form $\mathbb{C} / \mathfrak{a} \rightarrow \mathbb{C} / \mathfrak{a}^{-1}$, where $\mathfrak{l} \subset \mathcal{O}$ is an invertible prime ideal satisfying $N(\mathfrak{l}) \leq(\log p)^{2+\tilde{\varepsilon}}[\underline{6}, \S 3],[7$, , 13, $\S 2.1]$. Equivalently, $\mathcal{H}$ is the Cayley graph of the Picard group $\operatorname{Pic}(\mathcal{O})$ of the order $\mathcal{O}$ with respect to the generators $[\mathfrak{l}] \in \operatorname{Pic}(\mathcal{O})$, where $\mathfrak{l}$ ranges over the invertible prime ideals of $\mathcal{O}$ whose norm is at most $(\log p)^{2+\tilde{\varepsilon}}$. 


\subsection{The Spectral Gap of an Isogeny Graph}

For a particular isogeny graph $\mathcal{G}$ of ordinary elliptic curves, one can bound the nontrivial eigenvalues via character sum estimates under the Generalized Riemann Hypothesis. This is done via spectral analysis of the corresponding Cayley graph $\mathcal{H}$. For what follows, it will be convenient to view the eigenvectors of the adjacency matrix of $\mathcal{H}$ as functions on the corresponding ideal classes of the Picard group. The following proposition is proven in [13, §4]:

Proposition 5.3. Let $m=(\log p)^{2+\tilde{\varepsilon}}$ and let $e=\# \mathcal{O}^{\times}$.

(i) The graph $\mathcal{H}$ has eigenfunctions equal to the characters $\chi$ of $\operatorname{Pic}(\mathcal{O})$ with corresponding eigenvalues the character sums

$$
\lambda_{\chi}=\sum_{p \leq m} \sum_{\substack{\mathfrak{a} \subset \mathcal{O}, N \mathfrak{a}=p}} \chi(\mathfrak{a}) .
$$

(ii) Let $D<0$ and let $\mathcal{O}$ be an order of discriminant $D$. The trivial eigenvalue $\lambda_{\text {triv }}$ is equal to the number of ideal classes of the form $[\mathfrak{l}]$ where $\mathfrak{l}$ invertible prime ideal of $\mathcal{O}$ of norm at most $m$ (note that $\lambda_{\text {triv }}$ is asymptotically equal to $\frac{m}{e \log m}$ where $\left.e=\# \mathcal{O}^{\times}\right)$. If $\chi$ is a nontrivial character of the Picard group $\operatorname{Pic}(\mathcal{O})$, then under the Generalized Riemann Hypothesis,

$$
\lambda_{\chi}=O\left(m^{1 / 2} \log |m D|\right)
$$

Remark 5.1. Propositions 5.2 and 5.3 show the following: suppose that $S$ is a set of elliptic curves belonging to the same level of the isogeny volcano, such that $|\mathcal{G}| /|S|=(\log p)^{O(1)}$ and such that one can efficiently compute $\mathrm{DH}_{E, P}(u P, v P)$ for every $E \in S$ and every Diffie-Hellman triple $(P, u P, v P)$ for $E$. Then there is a random polynomial time reduction of the computation of the Diffie-Hellman function on an arbitrary curve $E \in V(\mathcal{G})$ to the Diffie-Hellman function on a curve in $S$. Hence, one can compute the Diffie-Hellman secret on any curve $E$ in $V(\mathcal{G})$ with high probability in time polynomial in $\log p$.

Indeed, a random walk of length polynomial in $\log p$ will connect $E$ to a curve in $S$ with high probability (high probability means $1-O\left(p^{-r}\right)$ for some $\left.r>0\right)$. Since any step in this random walk is an isogeny that is computable in time polynomial in $\log p$, the resulting composition of isogenies and their duals are computable in time polynomial in $\log p$ (even if the degree of the composition is large). Finally, if $(P, u P, v P)$ is a Diffie-Hellman triple for $E$ and $\phi: E \rightarrow E^{\prime}$ is an isogeny to an elliptic curve $E^{\prime} \in S$, one can consider the Diffie-Hellman triple $(\phi(P), u \phi(P), v \phi(P))$ on $E^{\prime}$ and compute the Diffie-Hellman function for that triple to obtain $u v \phi(P)$. After applying the dual isogeny, we obtain the point $d u v P$, where $d$ is the degree of the composition (note that the degree is polynomial in $\log p$ ). Finally, since we are in a prime-order subgroup, we compute $e$, such that de is congruent to 1 modulo the group order. The point $\operatorname{ed}(u v P)=$ $u v P$ is then the desired point. 
Remark 5.2. There exist isogeny graphs for supersingular elliptic curves as well. These supersingular graphs were first considered in [11] and [17. Their expansion properties were shown much later by Pizer [21, 22]. Given a prime $p$, the supersingular elliptic curves are always defined over $\mathbb{F}_{p^{2}}$. According to [17], all isomorphism classes of supersingular elliptic curves belong to the same isogeny class. In practice, we ignore supersingular curves in our argument for the main theorem. Yet, the corresponding isogeny graph is still an expander graphs.

\section{Random Self-reducibility}

We define random self-reducibility. Intuitively, we would like to prove that an efficient algorithm for the Diffie-Hellman function in the average case would imply an efficient algorithm in the worst case.

\subsection{Smooth Isogeny Classes and Random Self-reducibility}

Let $R$ be a fixed polynomial. Consider the following properties of a set $S$ of elliptic curves over $\mathbb{F}_{p}$ :

1. There exists a subset $S^{\prime} \subseteq S$ with $\left|S^{\prime}\right| /|S| \geq R(\log p)^{-1}$.

2. There exists an algorithm $\mathcal{A}$, such that: i) $\mathcal{A}$ computes the Diffie-Hellman function on any elliptic curve $E \in S^{\prime}$; ii) $\mathcal{A}$ produces random output whenever one feeds in a Diffie-Hellman triple for an elliptic curve $E \notin S^{\prime}$.

Definition 6.1. Let $S$ be a set of elliptic curves that satisfies conditions 1. and 2. We call $S$ random self-reducible with respect to $R$ if given an elliptic curve $E \in S$, one can compute the Diffie-Hellman function for any triple $(Q, u Q, v Q)$ on $E$ with expected $(\log p)^{O(1)}$ queries to $\mathcal{A}$ on elliptic curves $E^{\prime} \in S$ that are randomly distributed among all classes in $S$.

\subsection{Random Self-reducibility for Single Levels in the Isogeny Volcanoes}

We first show that horizontal levels in the isogeny volcanoes with sufficiently many curves on which the Diffie-Hellman problem is solvable are random selfreducible:

Lemma 6.1. Let $\mathcal{G}$ be the graph corresponding to a particular level of the isogeny volcano for some isogeny class of elliptic curves. Assume that the set of vertices $V(\mathcal{G})$ of $\mathcal{G}$ satisfies 1. and 2. for some polynomial $R$. Then $V(\mathcal{G})$ is random self-reducible with respect to $R$.

Proof. Let $E$ be any elliptic curve in $V(\mathcal{G})$ and $(P, u P, v P)$ be any Diffie-Hellman triple for $E$. We will show how to connect this input to the Diffie-Hellman function to an input on a random elliptic curve $E^{\prime}$ from $V(\mathcal{G})$ via a sequence of isogenies that are computable in polynomial time. Let $S^{\prime} \subset V(\mathcal{G})$ be the distinguished set from item 1 above. and let $\mu=\left|S^{\prime}\right| /|V(\mathcal{G})|$. Let $E_{0}=E$. We 
will use the fact that $\mathcal{G}$ is an expander graph. Let $\tau=\left\lfloor\frac{\log \left(\frac{2|V(\mathcal{G})|}{|S|^{1 / 2}}\right)}{\log \left(\nu^{-1}\right)}\right\rfloor+1$, where $\nu$ is the spectral gap for $\mathcal{G}$. Using the upper bound for $\nu$ from Proposition 5.2 we obtain that $\tau$ is polynomial in $\log p$, i.e., $\tau=(\log p)^{O(1)}$.

We repeat the following procedure $m \geq \frac{2}{\mu} \log p$ times:

1. Consider a random walk $E_{0}, E_{1}, \ldots, E_{\tau}$ on $\mathcal{G}$ of length $\tau$. Let $\phi$ be the composition of the isogenies along the walk, $\widehat{\phi}$ be the dual isogeny of $\phi$ and $d$ be their degree. Compute $e=d^{-1}$ modulo $q$ (recall that $q$ is the prime order of the original point $P$ ).

2. If $E^{\prime}=E_{\tau}$, query the oracle on the elliptic curve $E^{\prime}$ and the Diffie-Hellman triple $(\phi(P), u \phi(P), v \phi(P))$ under $\phi$.

3. If the oracle returns the point $Q$ on $E^{\prime}$, compute and return $e \widehat{\phi}(Q) \in E\left(\mathbb{F}_{p}\right)$.

Since the computation of a single isogeny of degree $\ell$ takes $O\left(\ell^{4}\right)$ time (see [14]), each of the above steps runs in time $O\left((\log p)^{8+4 \tilde{\varepsilon}} \tau\right)$ which is polynomial in $\log p$ (as do all other steps below).

By Proposition 5.2 the probability that $E_{\tau} \notin S^{\prime}$ is at most $1-\frac{\mu}{2}$. Thus, if we repeat the above steps $m$ times, the probability that none of the end points of the random walk is in $S^{\prime}$ is at most

$$
\left(1-\frac{\mu}{2}\right)^{m} \leq e^{-\frac{\mu m}{2}} \leq e^{-\frac{\mu \cdot 2 / \mu \log p}{2}}=O\left(p^{-1}\right) .
$$

Therefore, the above procedure will produce a list $A=L(P, u P, v P)$ of points that contains the desired point $u v P$ with high probability. To obtain the desired solution, we compute the list $B=L(P,(u+r) P, v P)$ for a random $r \in[1, q-1]$ as in the method of Shoup [23. We check if $A$ and $-r v P+B$ have a unique common element, and if so, we output it. Otherwise, we report a failure. The analysis of this last step is the same as in 23 .

\subsection{Random Self-reducibility for Multiple Levels in the Isogeny Volcanoes}

Owing to space limitations we will only outline how one can apply the methods of the single level case to solve the case of multiple levels in the isogeny volcano. Outside of this section, we restrict our discussion to the case of a single level.

Definition 6.2. Let $B$ be a positive real number. An isogeny class $S_{t}$ of elliptic curves is called B-smooth if its conductor $c_{t}$ is B-smooth, i.e., if any prime divisor of $c_{t}$ is at most $B$.

The next lemma proves reducibility of the Diffie-Hellman problem for a whole isogeny class (not just a single level). 
Lemma 6.2. Let $r>0$ be any real constant and assume that $S_{t}$ satisfies $\left.i\right)$ and ii) for some polynomial $R$, and that $S_{t}$ is $(\log p)^{r}$-smooth. Assuming the Generalized Riemann Hypothesis, any instance of the Diffie-Hellman problem on any elliptic curve $E \in S_{t}$ can be computed in time polynomial in $\log p$.

The next lemma guarantees that the conductor $c_{t}$ will have $O(\log \log p)$ distinct prime factors for almost all traces $t$ in the Hasse interval. Let $m$ be a positive integer such that $\log \log m>1$ and let $N_{m}$ be the number of traces $t \in H$, such that $c_{t}$ has less than $m$ distinct prime factors.

Lemma 6.3. There exists a constant $C$ (independent of $m$ and $p$ ) such that

$$
N_{m} \geq\left(1-e^{-C m \log m}\right)|H| \text {. }
$$

Proof omitted.

Remark 6.1. Suppose that $c>0$ is fixed. By choosing $k$ large enough (independent of $p$ ) and applying the above lemma for $m=k \log \log p$, we can guarantee that $N_{m}=\left(1-O\left((\log p)^{-c}\right)\right)|H|$. This means that for most of the traces $t \in H$, $c_{t}$ will have $O(\log \log p)$ distinct prime divisors.

For the classes $S_{t}$ for which the volcano has multiple levels, we may not be able to exploit random self-reducibility in some of them. We can bound $c_{t}$ to be small enough and having $O(\log \log p)$ prime divisors, so that starting from an arbitrary elliptic curve, we can reach the appropriate random self-reducible level in time polynomial in $\log p$ by searching through the levels via vertical isogenies and testing each level for random self-reducibility.

\section{Proof of Theorem 3.1}

\subsection{Notation}

Let $\mathcal{A}$ be the oracle from Theorem 3.1 and $\varepsilon$ be the corresponding advantage. A short Weierstrass equation $W$ is called LSB-predictable, if for any point $P \in$ $W\left(\mathbb{F}_{p}\right)$ of prime order $q>(\log p)^{2+\tilde{\varepsilon}}, \operatorname{Adv}_{W, P}(u P, v P)>\varepsilon$ (in other words, $\mathcal{A}$ predicts the least significant bit of the Diffie-Hellman function for $W$ and the generator $P$ with advantage $\varepsilon$ ).

More generally, if $T$ is any set of short Weierstrass equations over $\mathbb{F}_{p}$ and $0<\delta^{\prime}<1$ is a real number, we refer to $T$ as $\delta^{\prime}$-predictable if at least $\delta^{\prime}|T|$ elliptic curves in $T$ are LSB-predictable.

\subsection{Most of the Isogeny Classes Are Smooth}

Let $B$ be an arbitrary integer. The following lemma shows that almost all of the isogeny classes $S_{t}$ of elliptic curves over $\mathbb{F}_{p}$ are $B$-smooth. The latter will be useful in applying the tunneling argument and Lemma 6.2. 
Lemma 7.1. The number of traces $t \in H$, such that the isogeny class $S_{t}$ corresponding to $t$ is $B$-smooth is at least $\left(1-\frac{2}{B}\right)|H|$.

Proof. Fix a prime $\ell$, such that $B<\ell<\sqrt{p}$ and consider the solutions of the congruence

$$
t^{2} \equiv 4 p \quad \bmod \ell^{2}
$$

for $t \in H$. First, the congruence $t^{2} \equiv 4 p \bmod \ell$ has exactly $1+\left(\frac{4 p}{\ell}\right)$ solutions. Each such solution $t$ lifts uniquely to a solutions $\tilde{t}$ modulo $\ell^{2}$ by Hensel's lemma since the derivative of $f(x)=x^{2}-4 p$ does not vanish modulo $\ell>2$ at any such $t$. Thus,

$$
\begin{aligned}
\operatorname{Pr}_{t \in H}\left[c_{t} \text { is not } B-\text { smooth }\right] & \leq \sum_{B<\ell<\sqrt{p}} \frac{1}{\ell^{2}}\left[1+\left(\frac{4 p}{\ell}\right)\right]< \\
& <\sum_{B<\ell<\sqrt{p}} \frac{2}{\ell^{2}}<\int_{B}^{\infty} \frac{2}{u^{2}} d u=\frac{2}{B} .
\end{aligned}
$$

\subsection{Lower Bound on Smooth, Predictable Isogeny Classes}

Here, we show that there is a polynomial fraction of traces $t \in H$ such that $S_{t}$ is smooth and $C_{t}$ contains sufficiently many LSB-predictable short Weierstrass equations.

Lemma 7.2. Let $\delta$ and $c$ be as in the statement of Theorem 3.1. There exists a constant $c_{1}$ (independent of $p$ ), such that the number of traces $t \in H$ for which $S_{t}$ is $(\log p)^{c+2}$-smooth and $C_{t}$ is $\delta / 2$-predictable is at least $c_{1} \frac{|H|}{(\log p)^{c+1}(\log \log p)^{2}}$

Proof. Let

$$
S_{\delta / 2}=\left\{t \in H: C_{t} \text { is } \delta / 2 \text {-predictable }\right\}
$$

and

$$
U=\left\{t \in H \quad: \quad S_{t} \text { is }(\log p)^{c+2} \text {-smooth }\right\}
$$

By Lemma 7.1, $|U| \geq\left(1-\frac{2}{(\log p)^{c+2}}\right)|H|$. We would like to estimate $\left|U \cap S_{\delta / 2}\right|$. First, we need an estimate on $\left|S_{\delta / 2}\right|$. For each $t \in S_{\delta / 2}, C_{t}$ contains at most $\left|C_{t}\right|$ LSB-predictable curves. For each $t \notin S_{\delta / 2}, C_{t}$ contains at most $(\delta / 2)\left|C_{t}\right|$ LSBpredictable curves. Thus, we get the inequality

$$
\sum_{t \in S_{\delta / 2}}\left|C_{t}\right|+\sum_{t \notin S_{\delta / 2}} \frac{\delta}{2}\left|C_{t}\right| \geq|G|=\delta\left|\Gamma_{p}\right|
$$


We combine this with Lemma 4.1 to obtain

$$
\begin{aligned}
\delta\left|\Gamma_{p}\right| & \leq \sum_{t \in S_{\delta / 2}}\left|C_{t}\right|+\sum_{t \notin S_{\delta / 2}} \frac{\delta}{2}\left|C_{t}\right|=\sum_{t} \frac{\delta}{2}\left|C_{t}\right|+\sum_{t \in S_{\delta / 2}}\left(1-\frac{\delta}{2}\right)\left|C_{t}\right| \leq \\
& \leq \frac{\delta}{2}\left|\Gamma_{p}\right|+\left(1-\frac{\delta}{2}\right) c_{u}\left|S_{\delta / 2}\right| p^{3 / 2}(\log p)(\log \log p)^{2} .
\end{aligned}
$$

Thus,

$$
\left|S_{\delta / 2}\right| \geq\left(\frac{\delta / 2}{1-\delta / 2}\right) \frac{\left|\Gamma_{p}\right|}{c_{u} p^{3 / 2}(\log p)(\log \log p)^{2}} \geq c_{1}^{\prime} \frac{|H|}{(\log p)^{c+1}(\log \log p)^{2}},
$$

for some constant $c_{1}^{\prime}>0\left(\right.$ since $\left.\delta=O\left((\log p)^{c}\right)\right)$. Hence,

$$
\begin{aligned}
\left|U \cap S_{\delta / 2}\right| & =|U|+\left|S_{\delta / 2}\right|-\left|U \cup S_{\delta / 2}\right| \geq\left(1-\frac{2}{(\log p)^{c+2}}\right)|H|+ \\
& +c_{1}^{\prime} \frac{|H|}{(\log p)^{c+1}(\log \log p)^{2}}-|H| \geq c_{1} \frac{|H|}{(\log p)^{c+1}(\log \log p)^{2}}
\end{aligned}
$$

for some constant $c_{1}$ independent of $p$. This proves the lemma.

\subsection{Predicting LSB within an Isomorphism Class}

It was shown in [1] that within an isomorphism class of short Weierstrass equations, predicting the least significant bit on a non-negligible fraction of the short Weierstrass equations is at least as hard as computing the entire Diffie-Hellman secret key for the elliptic curve corresponding to this class.

For any short Weierstrass equation $W: y^{2}=x^{3}+a x+b$ and any $\lambda \in \mathbb{F}_{p}^{\times}$we denote by $W_{\lambda}$ the isomorphic curve $y^{2}=x^{3}+a \lambda^{4} x+b \lambda^{6}$ and by $\phi_{\lambda}: W \rightarrow W_{\lambda}$ the isomorphism $(x, y) \mapsto\left(\lambda^{2} x, \lambda^{3} x\right)$. The result is summarized as follows:

Theorem 7.1 (Boneh-Shparlinski). Let $0<\varepsilon, \delta<1$. Let $p$ be a prime and $W$ be a short Weierstrass equation over $\mathbb{F}_{p}$. Let $P \in W\left(\mathbb{F}_{p}\right)$ be a point of prime order. Suppose that there is a $\tau$-time algorithm $\mathcal{A}$, such that $\operatorname{Adv}_{W_{\lambda}, \phi_{\lambda}(P)}(\mathcal{A})>\varepsilon$ for at least $\delta$-fraction of all $\lambda \in \mathbb{F}_{p}^{\times}$. Then the Diffie-Hellman function for $W$ with respect to the generator $P$ can be computed in expected time $\tau \cdot\left(\varepsilon^{-1} \delta^{-1} \log p\right)^{O(1)}$.

\subsection{Predictable Isomorphism Classes within a Predictable Isogeny Class}

Lemma 7.3. Let $0<\beta<1$, such that $1 / \beta=O\left((\log p)^{c}\right)$, let $t \in H$ be a trace, such that $C_{t}$ be a $\beta$-predictable isogeny class of short Weierstrass equations. There exists a constant $0<c_{2}<1$, such that the number of $\beta / 2$-predictable isomorphism classes of elliptic curve inside $C_{t}$ is at least $c_{2} \frac{\left|S_{t}\right|}{(\log p)^{c}}$. 
Proof. Let $T_{\beta / 2}$ be the set of $\beta / 2$-predictable isomorphism classes of short Weierstrass models contained $C_{t}$. Each isomorphism class $I \subset C_{t}, I \in T_{\beta / 2}$ contains at most $|I|$ LSB-predictable elliptic curves and each isomorphism class $I \notin T_{\beta / 2}$ contains at most $\frac{\beta}{2}|I|$ LSB-predictable elliptic curves. Thus,

$$
\begin{aligned}
\beta\left|C_{t}\right| & \leq \sum_{\substack{I \subset C_{t}, I \in T_{\beta / 2}}}|I|+\sum_{\substack{I \subset C_{t}, I \notin T_{\beta / 2}}} \frac{\beta}{2}|I|=\sum_{I \subset C_{t}} \frac{\beta}{2}|I|+\sum_{\substack{I \subset C_{t}, I \in T_{\beta / 2}}}\left(1-\frac{\beta}{2}\right)|I| \leq \\
& \leq \frac{\beta}{2}\left|C_{t}\right|+\frac{(2-\beta) p}{4}\left|T_{\beta / 2}\right| .
\end{aligned}
$$

Therefore,

$$
\left|T_{\beta / 2}\right| \geq 2\left(\frac{\beta}{1-\beta / 2}\right) \frac{\left|C_{t}\right|}{p}>c_{2} \frac{|S|}{(\log p)^{c}},
$$

for some constant $c_{2}>0$ independent of $p\left(\right.$ since $\left.1 / \beta=O\left((\log p)^{c}\right)\right)$.

\subsection{Proof of Theorem 3.1}

Proof (Proof of Theorem 3.1). According to Lemma 7.2, there exists a constant $c_{1}$ (independent of $p$ ), such that for at least $c_{1} \frac{|H|}{(\log p)^{c+1}(\log \log p)^{2}}$ traces $t \in$ $H, S_{t}$ is $(\log p)^{c+2}$-smooth and $C_{t}$ is $\delta / 2$-predictable. Let $0<\mu<1$ be the real number defined by $2 \sqrt{p} \mu=\frac{c_{1}}{4} \cdot \frac{|H|}{(\log p)^{c+1}(\log \log p)^{2}}$. We will apply our refinement of Lenstra's lemma with this particular $\mu$. Indeed, let $\Sigma$ be the set of all traces $t \in H$ which satisfy $|t| \leq 2 \sqrt{p}(1-\mu)$ and such that $S_{t}$ is $(\log p)^{c+2}$ smooth and $C_{t}$ is $\delta / 2$-predictable. Then

$$
|\Sigma| \geq\left\lceil\frac{c_{1}}{2} \cdot \frac{|H|}{(\log p)^{c+1}(\log \log p)^{2}}\right\rceil .
$$

Since we have assumed the Generalized Riemann Hypothesis, Proposition 4.1(ii) implies that

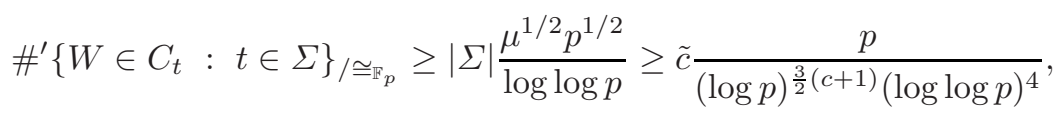

for some constant $\tilde{c}$ independent of $p$. Let

$$
S:=\left\{W \in C_{t}: t \in \Sigma\right\} / \simeq_{\mathbb{F}_{p}}
$$

Since the weighted cardinality of each isogeny class is $p / 2, p / 4$ or $p / 6$, we obtain that there exists a constant $\tilde{c}^{\prime}$ (independent of $p$ ), such that

$$
|S| \geq \tilde{c}^{\prime} \frac{\left|\Omega_{p}\right|}{(\log p)^{\frac{3}{2}(c+1)}(\log \log p)^{4}} .
$$


We claim that $S$ satisfies the properties of the theorem. Indeed, by Lemma 7.3 applied to $\beta=\delta / 2$ we obtain that for each $t \in \Sigma, C_{t}$ contains a polynomial fraction of $\delta / 4$-predictable isomorphism classes. The result of Boneh and Shparlinski then implies that one can compute the Diffie-Hellman function on each of these isomorphism classes in time $\tau(\log p)^{O(1)}($ since $1 / \delta$ is polynomial in $\log p$ ). Finally, applying Lemma 6.2 we obtain that one can solve the Diffie-Hellman problem on any $E \in S$ in time $\tau(\log p)^{O(1)}$. That completes the proof.

Acknowledgements. We thank Dan Boneh, David Jao, Steve Miller, Bjorn Poonen and Ken Ribet for discussions.

\section{References}

1. Boneh, D., Shparlinski, I.: On the unpredictability of bits of elliptic curve DiffieHellman scheme. In: Kilian, J. (ed.) CRYPTO 2001. LNCS, vol. 2139, pp. 201-212. Springer, Heidelberg (2001)

2. Boneh, D., Venkatesan, R.: Hardness of computing the most significant bits of secret keys in Diffie-Hellman and related schemes. In: Koblitz, N. (ed.) CRYPTO 1996. LNCS, vol. 1109, pp. 129-142. Springer, Heidelberg (1996)

3. Cohen, H., Frey, G. (eds.): Handbook of elliptic and hyperelliptic curve cryptography, Theory and Practice (2005)

4. Deuring, M.: Die Typen der Multiplikatorenringe elliptischer Funktionenkörpen, vol. 14, pp. 197-272. Abh. Math. Sem. Hansischen Univ (1941)

5. Fouquet, M., Morain, F.: Isogeny volcanoes and the SEA algorithm. In: Fieker, C., Kohel, D.R. (eds.) ANTS 2002. LNCS, vol. 2369, pp. 276-291. Springer, Heidelberg (2002)

6. Galbraith, S.D.: Constructing isogenies between elliptic curves over finite fields. LMS J. Comput. Math. 2, 118-138 (1999) (electronic)

7. Galbraith, S.D., Hess, F., Smart, N.P.: Extending the GHS Weil descent attack. In: Knudsen, L.R. (ed.) EUROCRYPT 2002. LNCS, vol. 2332, pp. 29-44. Springer, Heidelberg (2002)

8. Gonzalez Vasco, M.I., Shparlinski, I.: Security of the most significant bits of the Shamir message passing scheme. Math. Comput. 71(237), 333-342 (2002)

9. Gross, B.H.: Heights and the special values of $L$-series, Number theory (Montreal, Que., 1985). In: CMS Conf. Proc., vol. 7, pp. 115-187. Amer. Math. Soc., Providence (1987)

10. Howgrave-Graham, N., Nguyen, P.Q., Shparlinski, I.: Hidden number problem with hidden multipliers, timed-release crypto, and noisy exponentiation. Math. Comput. 72(243), 1473-1485 (2003)

11. Ihara, Y.: Discrete subgroups of $\mathrm{PL}\left(2, k_{\wp}\right)$, Algebraic Groups and Discontinuous Subgroups. In: Proc. Sympos. Pure Math., Boulder, Colo., 1965, vol. IX, pp. 272 278. Amer. Math. Soc., Providence (1966)

12. Jao, D., Jetchev, D., Venkatesan, R.: On the security of certain partial DiffieHellman secrets. In: Srinathan, K., Pandu Rangan, C., Yung, M. (eds.) INDOCRYPT 2007. LNCS, vol. 4859. Springer, Heidelberg (2007)

13. Jao, D., Miller, S.D., Venkatesan, R.: Do all elliptic curves of the same order have the same difficulty of discrete log? In: Roy, B. (ed.) ASIACRYPT 2005. LNCS, vol. 3788, pp. 21-40. Springer, Heidelberg (2005) 
14. Kohel, D.: Endomorphism rings of elliptic curves over finite fields. University of California, Berkeley, Ph.D. thesis (1996)

15. Lenstra, H.W.: Factoring integers with elliptic curves. Ann. of Math 126(2), 649$673(1987)$

16. Menezes, A.J., van Oorschot, P.C., Vanstone, S.A.: Handbook of applied cryptography. CRC Press, Inc., Boca Raton (1996)

17. Mestre, J.-F.: La méthode des graphes. Exemples et applications. In: Proceedings of the international conference on class numbers and fundamental units of algebraic number fields (Katata), pp. 217-242 (1986)

18. Nguyen, P.Q.: The dark side of the hidden number problem: Lattice attacks on DSA. In: Proc. Workshop on Cryptography and Computational Number Theory, pp. 321-330 (2001)

19. Nguyen, P.Q., Shparlinski, I.: The insecurity of the digital signature algorithm with partially known nonces. J. Cryptology 15(3), 151-176 (2002)

20. Nguyen, P.Q., Shparlinski, I.: The insecurity of the elliptic curve digital signature algorithm with partially known nonces. Des. Codes Cryptography 30(2), 201-217 (2003)

21. Pizer, A.K.: Ramanujan graphs and Hecke operators. Bull. Amer. Math. Soc (N.S.) 23(1), 127-137 (1990)

22. Pizer, A.K.: Ramanujan graphs, Computational perspectives on number theory (Chicago, IL, 1995). In: AMS/IP Stud. Adv. Math., vol. 7, pp. 159-178. Amer. Math. Soc., Providence (1998)

23. Shoup, V.: Lower bounds for discrete logarithms and related problems. In: Fumy, W. (ed.) EUROCRYPT 1997. LNCS, vol. 1233, pp. 256-266. Springer, Heidelberg (1997)

24. Shparlinski, I.: On the generalized hidden number problem and bit security of XTR. In: Bozta, S., Sphparlinski, I. (eds.) AAECC 2001. LNCS, vol. 2227, pp. 268-277. Springer, Heidelberg (2001)

25. Shparlinski, I.: Cryptographic applications of analytic number theory: Complexity lower bounds and pseudorandomness. PCS, vol. 22. Birkhäuser, Basel (2003)

26. Shparlinski, I., Winterhof, A.: A hidden number problem in small subgroups. Math. Comp. 74, 2073-2080 (2005)

27. Silverman, J.H.: The arithmetic of elliptic curves. Springer, New York (1992)

28. Tate, J.: Endomorphisms of abelian varieties over finite fields. Invent. Math. 2, 134-144 (1966) 